\title{
Management of recurrent ureteral stricture: A retrospectively comparative study with robot-assisted laparoscopic surgery versus open approach
}

\author{
Qing Wang ${ }^{1}$, Yuchao Lu ${ }^{1}$, Henglong Hu ${ }^{1}$, Jiaqiao Zhang ${ }^{1}$, Baolong Qin ${ }^{1}$, Jianning Zhu ${ }^{1}$, Najib Isse Dirie ${ }^{1}$,

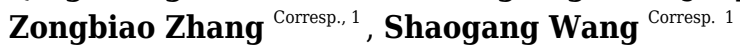 \\ ${ }^{1}$ Department of Urology, Tongji Hospital, Tongji Medical College, Huazhong University of Science and Technology, Wuhan, Hubei, China \\ Corresponding Authors: Zongbiao Zhang, Shaogang Wang \\ Email address: zzb070@126.com, sgwangtjm@163.com
}

Background: Management of recurrent ureteral stricture is challenging. Consensus on the best surgical choice has not been demonstrated. In this study, we aim to report our experience in treating recurrent ureteral stricture and demonstrate whether robot-assisted procedure for redo ureteral surgery is as effective as open procedure while remaining less invasive.

Methods: We retrospectively assessed 41 patients (22 robot-assisted surgeries and 19 open surgeries) who underwent consecutive robot-assisted and open procedures for redo ureteral surgery from January 2014 to January 2018 in our institution. Perioperative outcomes, including demographics, operative time, estimated blood loss, complications, pain scores, success rate and cost, were compared between two groups.

Results:There was no significant intergroup difference in terms of age, body mass index, gender composition and American Society of Anesthesiologists (ASA) scores. Thirty one patients underwent redo pyeloplasty and ten underwent redo uretero-ureterostomy. Compared with open group, robot-assisted group showed shorter operative time $(124.55 \mathrm{~min}$ vs. $185.11 \mathrm{~min}, p<0.0001)$, less estimated blood loss ( $100.00 \mathrm{~mL}$ vs $182.60 \mathrm{~mL}, p=0.008)$, and higher cost (61161.77¥ vs 39470.79¥, $p<0.0001$ ). Complication rate and pain scores were similar between two groups. Median follow-up periods were 30 and 48 months for robot-assisted and open group respectively. Success rate in the robot-assisted (85.71\%) and the open group (82.35\%) was not significantly different.

Conclusions: Robot-assisted surgery for recurrent stricture after previous ureteral reconstruction is as effective as open procedure and is associated with shorter operative time and less estimated blood loss. 
1 Management of recurrent ureteral stricture: A retrospectively comparative study with

2

3 Qing Wang, Yuchao Lu, Henglong Hu, Jiaqiao Zhang, Baolong Qin, Jianning Zhu, Najib Isse

4 Dirie, Zongbiao Zhang*, Shaogang Wang*.

5

6 Affiliations:

7 Department of Urology, Tongji Hospital, Tongji Medical College, Huazhong University of

8 Science and Technology, Wuhan, Hubei, 430030, China

\section{9}

10

11

12

13

14

15

16

17

18

19

20

21

22

23

24

25

26

27

E-mails:

Qing Wang: wangqingtjm@hust.edu.cn

Yuchao Lu: luyuchao@hust.edu.cn

Henglong Hu: huhenglong@hust.edu.cn

Jiaqiao Zhang: medzjq@163.com

Baolong Qin: 771747457@qq.com

Jianning Zhu: zhu_jianning@163.com

Najib Isse Dirie: najiibcd@hotmail.com

\section{* Co-Corresponding authors:}

Zongbiao Zhang

Email: zzb070@126.com

Shaogang Wang

Email: sgwangtjm@163.com 
Background: Management of recurrent ureteral stricture is challenging. Consensus on the best surgical choice has not been demonstrated. In this study, we aim to report our experience in treating recurrent ureteral stricture and demonstrate whether robot-assisted procedure for redo ureteral surgery is as effective as open procedure while remaining less invasive.

37

Methods: We retrospectively assessed 41 patients (22 robot-assisted surgeries and 19 open surgeries) who underwent consecutive robot-assisted and open procedures for redo ureteral surgery from January 2014 to January 2018 in our institution. Perioperative outcomes, including demographics, operative time, estimated blood loss, complications, pain scores, success rate and cost, were compared between two groups.

Results: There was no significant intergroup difference in terms of age, body mass index, gender composition and American Society of Anesthesiologists (ASA) scores. Thirty one patients underwent redo pyeloplasty and ten underwent redo uretero-ureterostomy. Compared with open group, robot-assisted group showed shorter operative time $(124.55 \mathrm{~min}$ vs. $185.11 \mathrm{~min}, \mathrm{p}<0.0001)$, less estimated blood loss $(100.00 \mathrm{~mL}$ vs $182.60 \mathrm{~mL}, \mathrm{p}=0.008)$, and higher cost $(61161.77 ¥$ vs 39470.79¥, $\mathrm{p}<0.0001)$. Complication rate and pain scores were similar between two groups. Median follow-up periods were 30 and 48 months for robot-assisted and open group respectively. Success rate in the robot-assisted $(85.71 \%)$ and the open group $(82.35 \%)$ was not significantly different.

Conclusions: Robot-assisted surgery for recurrent stricture after previous ureteral reconstruction 
55

56

57

58

59

60

61

62

63

64

65

66

67

is as effective as open procedure and is associated with shorter operative time and less estimated blood loss.

5

58

9

(6) Management of recurrent stricture after previous ureteral reconstruction is challenging, both in terms of decision-making and surgical technique. Endopyelotomy is the most minimally invasive endourological choice for recurrent ureteropelvic junction obstruction (UPJO). However, the reported success rate (39\%-83.5\%) seems to be suboptimal. [1-3] Hence, there comes again an interest in secondary reconstruction. Redo ureteral surgery is difficult due to scar formation, altered anatomic planes and decreased vascularity of the ureter. In such cases, open surgery is an excellent choice and the success rate of open redo pyeloplasty reaches up to $80 \%-100 \%$. [4-6] Open procedure has been then suggested as the gold standard for recurrent UPJO. Redo laparoscopic pyeloplasty also shows comparable outcomes to open procedure. [7, 8] However, laparoscopic procedure is more technically difficult and requires a longer learning curve, which limits its widespread use in redo ureteral surgery.

The da Vinci robot assisted system, which shows advantages of three-dimensional vision, tremor 3 filtering and seven degrees of freedom, has been increasingly used in ureteral reconstruction. [9, 74 10] In a large series conducted by Buffi et al., they find that robotic surgery for benign ureteral 75 stricture and UPJO is associated with low risk of high-grade complications and good outcomes. 76 [11] Subsequently, more and more studies have demonstrated that robot-assisted redo 77 pyeloplasty is minimally invasive and effective for recurrent UPJO, with excellent success rate 78 ranging from $88 \%$ to $100 \%$. [12-14]

80 To the best of our knowledge, there has been no study comparing the outcomes of robot-assisted 81 procedure with open procedure in redo ureteral surgery. Moreover, most previous studies focus 
82

83

84

on pediatric failed pyeloplasty, while adult cases and cases involving recurrent stricture in other sites of the ureter are rarely reported. In the present study, outcomes of robotic and open procedure in redo pyeloplasty and uretero-ureterostomy were compared. We aim to report our experience in treating recurrent ureteral stricture and provide urologists with some evidence for surgical decision.

\section{Methods \& Materials}

We retrospectively identified consecutive robot-assisted and open procedures performed from January 2014 to January 2018 in our institution for recurrent stricture with previous ureteral reconstruction. Patients treated with a "simple" redo ureteral anastomosis were included. Preoperative clinical assessments included evaluation of symptoms and computed tomography (CT), urography, magnetic resonance urography or intravenous urography. Indications for surgery included persistent clinical symptoms or worsening hydronephrosis on imaging or with worsening renal function. All robot-assisted procedures were performed by one experienced urologist (WSG) using the da Vinci Surgical System (Intuitive Surgical, Sunnyvale, CA). Open surgeries were performed by two experienced surgeons (including WSG). The choice between a robot-assisted and an open approach was based on the surgeon's preference and the patient's choice. Follow-up assessments were conducted in the third and sixth months after removing double $\mathrm{J}$ stent and then once every year. Success was defined as a stable or reduced degree of hydronephrosis on ultrasound or CT with symptom relief. Complications were classified using the Clavien-Dindo grading system. [15] Perioperative outcomes, including operative time, estimated blood loss, complications, pain scores, cost, and success rate were compared between two groups.

This study has been approved by the Institutional Review Board of Tongji Hospital, Tongji Medical College, Huazhong University of Science and Technology (2019S935). Absence of informed consent was approved because of the retrospective nature of the study. In addition, the 
109

110

\section{Procedures}

112 Patients underwent dismembered pyeloplasty for UPJO and uretero-ureterostomy for proximal 113

data were analyzed anonymously. ureter strictures (ureteral pelvic junction to the external iliac vessels). All robotic procedures were performed transperitoneally. Patients were placed in a modified 45-degree lateral decubitus position after general anesthesia. A periumbilical 12-mm camera port, two 8-mm robotic trocars oriented toward the stricture and two 12-mm assistant ports were placed for the operation (Figure 1). Iliac vessels, gonadal veins and paracolic sulcus were all important anatomical markers to quickly identify ureter or renal pelvis. The stricture segment was identified according to preoperative imaging localization and intraoperative morphological changes (dilated to normal ureter). After excising the stricture segment, proximal ureter was incised along the longitudinal axis of ureter to renal pelvis or at least $1 \mathrm{~cm}$ normal ureter was visualized. The normal distal ureter was also incised for $1 \mathrm{~cm}$. Once both ends of the ureter were adequately trimmed to healthy areas and correctly oriented, end-to-end reanastomosis was started laterally and performed in an interrupted fashion with a 4-0 polyglycolic acid suture. A double J ureteral stent was normally placed in our institution for about 4 weeks and a drain tube was also placed. The Foley catheter was placed for 2 to 3 days after surgery and the drainage tube was removed on the following day if there was no increase in drain output.

Surgical details of open procedure have been previously described by other investigators. [16] General principles were similar to those described for the robot-assisted procedure.

\section{Statistics}

Statistical analysis was performed with SPSS for Windows version 19.0 (IBM Corporation, Armonk, NY). For variables with normal distribution, data were presented as mean \pm standard deviation (SD) and compared using Student's t-test. For variables with a non-normal distribution, 
136

137

138

139

140

141

142

143

144

145

146

147

148

149

150

151

152

153

154

155

156

157

158

159

160

161

162

data were presented as median (range) and were compared using the Mann-Whitney U-test. Categorical variables were compared by Chi-squared test or Fisher's exact test.

\section{Results}

A total of 41 patients underwent redo ureteral reconstruction in our institution, including 22 robot-assisted and 19 open cases. Most patients came to hospital with flank pain while some recurrence was asymptomatic. Three patients presented with fever due to urinary infection. Hematuria was detected in one patient and the stricture was confirmed to be benign with pathological assessment. Initial etiologies for ureteral stricture were clarified into congenital, stone disease, vascular anomalies and polyp. Median interval between initial and redo surgery was 8 years (range, 1 to 25 years) in the robot-assisted group and 8 years (range, 0.5 to 31 years) in the open group. Primary surgery for ureteral stricture consisted of 27 open procedures and 14laparoscopic procedures. Missed lower pole crossing vessels in initial surgery were found in two patients. Some patients had once chosen endourological techniques after failed initial surgery, such as double $\mathrm{J}$ stent, balloon dilation and endopyelotomy. Most (80.9\%) patients directly underwent redo surgery. Moreover, one patient in the open group had undergone two open reconstructions previously (Table 1).

No significant difference was found in terms of age, body mass index (BMI), gender composition, disease side and American Society of Anesthesiologists (ASA) scores between two groups. In our series, 19 patients underwent redo pyeloplasty for recurrent UPJO and three patients underwent redo uretero-ureterostomy for recurrent proximal ureteral stricture with robotic procedure. None of the robot-assisted operations necessitated an open conversion. Correspondingly, 12 patients underwent redo pyeloplasty and seven patients underwent redo uretero-ureterostomy in the open group. There were no intraoperative complications in both groups. We encountered many challenges during dissection of periureteral planes due to significant fibrosis and adhesions in most patients. Compared with open group, robotic group 
163

164

165

166

167

168

169

170

171

172

173

174

175

176

177

178

179

180

181

182

183

184

185

186

187

188

189

showed shorter operative time $(124.55 \mathrm{~min}$ vs. $185.11 \mathrm{~min}, \mathrm{p}<0.0001)$ and less estimated blood loss $(100.00 \mathrm{~mL}$ vs. $182.6 \mathrm{~mL}, \mathrm{p}=0.008)$. Postoperative pain was assessed on the first day using visual analogue scores (VAS) and no significant difference was observed in the pain scores between two groups. Postoperative complication rate was similar between two groups. Although more patients suffered from fever (Grade II) in the open group, no significant difference was detected (36.84\% vs. 9.09\%, p=0.057). One patient suffered leakage of urine (Grade IIIa) and one showed incision hematoma (Grade IIIb) after open surgery. The patient with urine leakage experienced longer drainage time and longer double $\mathrm{J}$ insertion. The other patient underwent surgical intervention for the incision hematoma. In addition, robot-assisted group required higher cost (61161.77¥ vs 39470.79¥, p<0.0001). (Table 2)

\section{Follow up}

One patient in robot-assisted group and two in open group were lost to follow-up. The median follow-up duration was 30 months (range, 19-48 months) for the robot-assisted group and 48 months (range, 20-63 months) for the open group. There is significant difference in terms of follow-up length between two groups $(p=0.001)$. Two patients in the open group showed more severe hydronephrosis in the sixth and twelfth months after surgery. Both of them were treated with double $\mathrm{J}$ stent placement but outcomes were not ideal. One patient underwent nephrectomy and the other underwent dialysis. For patients with stable hydronephrosis, we assessed their renal function via routine biochemical analysis and all of them showed stable renal function. The success rate in the robot-assisted and open groups was $85.71 \%$ and $82.35 \%$ respectively, with no statistical difference between two groups (Table 3).

\section{Discussion}

It is reported that approximately $11 \%$ of the children who underwent pyeloplasty require a secondary procedure in the United States. [17] Signs of recurrent obstruction are variable. Jacobson et al. report that $36 \%$ patients with recurrent UPJO showed symptoms of pain, urinary 
190

191

192

193

194

195

196

197

198

199

200

201

202

203

204

205

206

207

208

209

210

211

212

213

214

215

216

infection or hematuria in their series. [18] In our study, 56.09\% patients came to the hospital with flank pain while $34.15 \%$ without symptoms and just showed a progressive hydronephrosis. Recurrence appears early after the first intervention in many studies. However, recurrence might occur until several years after primary repair in our series, indicating a need for long-term follow-up of these patients. We found that the most common cause for recurrent stricture was periureteric fibrosis or scars caused by urinary extravasation, which restricted peristalsis of the ureter. Missed lower pole crossing vessels was also another reason for failed pyeloplasty. In an anatomic analysis of 146 endocasts of kidney collecting system together with intrarenal arteries and veins, Sampaio and Favorito found that $65.1 \%$ of the cases showed renal vessels on the anterior surface of the ureteropelvic junction. [19] When pyeloplasty is done via retroperitoneal approach, there is a limited view of the anterior surface and the anterior crossing vessels might be missed.

Studies have shown that open redo pyeloplasty is associated with excellent outcomes for recurrent stricture. Redo laparoscopic pyeloplasty is also an effective treatment for recurrent UPJO, with reported success rate of $80 \%-100 \%$. [7, 8] Abdel-Karim et al demonstrate similar success rate between open and laparoscopic redo pyeloplasty. Although laparoscopic technique is associated with shorter hospital stays and less postoperative pain, it is more difficult and involves longer operative time and more blood loss. [4] Similarly, Chandru et al. and Clayman et al. both report in their early series that redo laparoscopic pyeloplasty requires a long operative time (average $6.2 \mathrm{~h}$ and $9 \mathrm{~h}$ respectively). [20, 21]

Introduction of robotic systems has overcome limitations of laparoscopy. Robot-assisted redo pyeloplasty has been reported to be an excellent choice for recurrent UPJO. Niver et al. report that robotic reoperation is as safe and effective as primary operation for UPJO. [22] Atug et al. note a success rate of $100 \%$ in one of the first pediatric series of robot-assisted redo pyeloplasty. [23] Lindgren et al. conduct robot-assisted redo pyeloplasty in 16 pediatric patients and $88 \%$ 
217 patients show postoperative improvement on radiographic findings. [12] Helmal et al. (9 patients) 218 also report a success rate of $100 \%$ for redo robot-assisted pyeloplasty. [24] Correspondingly, we 219 achieved a success rate of $85.71 \%$ for robot-assisted procedure in the present study. Three 220 patients showed stable hydronephrosis and unrelieved symptoms after robot-assisted surgery, but 221 their renal function remained stable during the follow-up period. No patient in the robot-assisted 222 group required further intervention.

223

To our knowledge, this study represented the first attempt to compare the outcomes of robot-

225

226

227

228

229

230

231

232

233

234

235

236

237

238

239

240

241

242

243 assisted and open procedure in treating recurrent benign ureteral stenosis of the UPJ and the ureter. We found that robotic assistance significantly reduced the operative time and estimated blood loss for redo ureteral surgeries, which showed advantages of minimal invasion. Benefits of robotic assistance were seen in easier meticulous dissection, better delineation of previous scarred tissue and preservation of the periureteral sheath containing blood supply to ureter. Lee et al. demonstrate shorter hospital stay and similar success rate for robot-assisted procedure compared to open procedure in treating primary UPJO. They also indicate that although robotassisted procedure requires longer operative time (219min vs. 181min), it improves and approaches the operative time of open procedure as the operator's experience increases. [25] Isac and Kozinn et al. also reported similar findings in their experience with robotic and open ureteroneocystostomy. [26, 27]

No significant difference in terms of postoperative complication rate between robotic and open group was observed in our study, which is similar with what Abdel-Karim et al. find in their series of laparoscopic versus open redo pyeloplasty. [4] This may be partly due to the small sample size in our study. However, it should be indicated that more patients suffered fever, and urine leakage and incision hematoma were observed in the open group, indicating a more invasive character of the open procedure. One feature of robot-assisted surgery observed in our study was that this technique was more expensive. Patients in robot-assisted group spent an 
244 average of $20,000 ¥$ more than those in open group and this may influence the choice of surgical 245 approach among patients.

246

247 There are several limitations in our study. Firstly, it is a relatively small retrospective case series 248 with selection bias. A larger number of patients should be involved in the future to verify current 249 findings. Secondly, we don't conduct renal emission computed tomography to assess the affected 250 side renal function in all patients. Thirdly, there is significant difference in terms of follow-up 251 length between robotic and open group, a longer follow-up time is required to confirm the 252 success rate.

253

\section{Conclusions}

255 Robot-assisted procedure for recurrent stricture after previous ureteral reconstruction is as 256 effective as open procedure. Moreover, robot-assisted technique shows decreased blood loss and 257 less operative time, which provides advantages of minimally invasive surgery.

259 Abbreviations:

260 UPJO: Ureteropelvic junction obstruction

261 CT: Computed tomography

262 BMI: Body mass index

263 VAS: Visual analogue scores

264 ASA: American society of anesthesiologists

265

266 Funding

267 This work was supported by National Key Research and Development Program of China 268 (2016YFC0902601).

269

270 Conflicts of interest 
271 The authors declare that there is no conflict of interest regarding the publication of this paper.

272

\section{Acknowledgements}

274 Not applicable.

275

276

277

278

279

280

281

282

283

284

285

286

287

288

289

290

291

292

293

1. Braga LH, Lorenzo AJ, Skeldon S, Dave S, Bagli DJ, Khoury AE, Pippi Salle JL, Farhat WA. Failed pyeloplasty in children: comparative analysis of retrograde endopyelotomy versus redo pyeloplasty. J Urol 2007; 178: 2571-2575.

2. Patel T, Kellner CP, Katsumi H, Gupta M. Efficacy of endopyelotomy in patients with secondary ureteropelvic junction obstruction. J Endourol 2011; 25: 587-591.

3. Abdrabuh AM, Salih EM, Aboelnasr M, Galal H, El-Emam A, El-Zayat T. Endopyelotomy versus redo pyeloplasty for management of failed pyeloplasty in children: A single center experience. J Pediatr Surg 2018; 53: 2250-2255.

4. Abdel-Karim AM, Fahmy A, Moussa A, Rashad H, Elbadry M, Badawy H, Hammady A. Laparoscopic pyeloplasty versus open pyeloplasty for recurrent ureteropelvic junction obstruction in children. J Pediatr Urol 2016; 12: 401.e1-e6.

5. Piaggio LA, Noh PH, González R. Reoperative laparoscopic pyeloplasty in children: comparison with open surgery. J Urol 2007; 177: 1878-1882.

6. Vannahme M, Mathur S, Davenport K, Timoney AG, Keeley FX Jr. The management of secondary pelvi-ureteric junction obstruction - a comparison of pyeloplasty and endopyelotomy. BJU Int 2014; 113: 108-112.

7. Moscardi PR, Barbosa JA, Andrade HS, Mello MF, Cezarino BN, Oliveira LM, Srougi M, Dénes FT, Lopes RI. Reoperative laparoscopic ureteropelvic junction obstruction repair in children: safety and efficacy of the technique. J Urol 2017; 197: 798-804.

8. Powell C, Gatti JM, Juang D, Murphy JP. Laparoscopic pyeloplasty for ureteropelvic junction obstruction following open pyeloplasty in children. J Laparoendosc Adv Surg Tech A 2015; 25: 
298

299

300

301

302

303

304

305

306

307

308

309

310

311

312

313

314

315

316

317

318

319

320

321

322

323

324

858-863.

9. Minnillo BJ, Cruz JA, Sayao RH, Passerotti CC, Houck CS, Meier PM, Borer JG, Diamond DA, Retik AB, Nguyen HT. Long-term experience and outcomes of robotic assisted laparoscopic pyeloplasty in children and young adults. J Urol 2011; 185: 1455-60.

10. Di Gregorio M, Botnaru A, Bairy L, Lorge F. Passing from open to robotic surgery for dismembered pyeloplasty: a single centre experience. Springerplus 2014; 3: 580.

11. Buffi NM, Lughezzani G, Hurle R, Lazzeri M, Taverna G, Bozzini G, Bertolo R, Checcucci E, Porpiglia F, Fossati N, Gandaglia G, Larcher A, Suardi N, Montorsi F, Lista G, Guazzoni G, Mottrie A. Robot-assisted surgery for benign ureteral strictures: experience and outcomes from four tertiary care institutions. Eur Urol 2017; 71: 945-951.

12. Lindgren BW, Hagerty J, Meyer T, Cheng EY. Robot-assisted laparoscopic reoperative repair for failed pyeloplasty in children: a safe and highly effective treatment option. J Urol 2012; 188: 932-937.

13. Asensio M, Gander R, Royo GF, Lloret J. Failed pyeloplasty in children: is robot-assisted laparoscopic reoperative repair feasible? J Pediatr Urol 2015; 11: 69.e1-e6.

14. Davis TD, Burns AS, Corbett ST, Peters CA. Reoperative robotic pyeloplasty in children. J Pediatr Urol 2016; 12: 394.e1-e7.

15. Dindo D, Demartines N, Clavien PA. Classification of surgical complications: a new proposal with evaluation in a cohort of 6336 patients and results of a survey. Ann Surg 2004; 240: $205-213$.

16. Stephen Y. Nakada, Sara L. Best: Management of upper urinary tract obstruction. In: Campbell-Walsh urology, 11th ed. Philadelphia, PA, USA: Elsevier, 2016, pp. 1115-1117.

17. Dy GW, His RS, Holt SK, Lendvay TS, Gore JL, Harper JD. National trends in secondary procedures following pediatric pyeloplasty. J Urol 2016; 195: 1209-1214.

18. Jacobson DL, Shannon R, Johnson EK, Gong EM, Liu DB, Flink CC, Meyer T, Cheng EY, Lindgren BW. Robot-assisted laparoscopic reoperative repair for failed pyeloplasty in children: an updated series. J Urol 2019; 201: 1005-1011. 
325

326

327

328

329

330

331

332

333

334

335

336

337

338

339

340

341

342

343

344

345

346

347

19. Sampaio FJ, Favorito LA. Ureteropelvic junction stenosis: vascular anatomical background for endopyelotomy. J Urol 1993; 150: 1787-1791.

20. Sundaram CP, Grubb RL 3rd, Rehman J, Yan Y, Chen C, Landman J, McDougall EM, Clayman RV. Laparoscopic pyeloplasty for secondary ureteropelvic junction obstruction. J Urol 2003; 169: 2037-2040.

21. Nakada SY, McDougall EM, Clayman RV. Laparoscopic pyeloplasty for secondary ureteropelvic junction obstruction: preliminary experience. Urology 1995; 46: 257-260.

22. Niver BE, Agalliu I, Bareket R, Mufarrij P, Shah O, Stifelman MD. Analysis of roboticassisted laparoscopic pyleloplasty for primary versus secondary repair in 119 consecutive cases. Urology 2012; 79: 689-694.

23. Atug F, Burgess SV, Castle EP, Thomas R. Role of robotics in the management of secondary ureteropelvic junction obstruction. Int J Clin Pract 2006; 60: 9-11.

24. Hemal AK, Mishra S, Mukharjee S, Suryavanshi M. Robot assisted laparoscopic pyeloplasty in patients of ureteropelvic junction obstruction with previously failed open surgical repair. Int J Urol 2008; 15: 744-746.

25. Lee RS, Retik AB, Borer JG, Peters CA. Pediatric robot assisted laparoscopic dismembered pyeloplasty: comparison with a cohort of open surgery. J Urol 2006; 175: 683-687.

26. Kozinn SI, Canes D, Sorcini A, Moinzadeh A. Robotic versus open distal ureteral reconstruction and reimplantation for benign stricture disease. J Endourol 2012; 26: 147-151.

27. Isac W, Kaouk J, Altunrende F, Rizkala E, Autorino R, Hillyer SP, Laydner H, Long JA, Kassab A, Khalifeh A, Panumatrassamee K, Eyraud R, Falcone T, Haber GP, Stein RJ. Robot-assisted ureteroneocystostomy: technique and comparative outcomes. J Endourol 2013; 27 : $318-323$. 


\section{Table 1 (on next page)}

Description of symptoms, recurrent interval, etiology for initial stricture, and primary procedures for patients. 
Table 1.Description of symptoms, recurrent interval, etiology for initial stricture, and primary procedures for patients.

\begin{tabular}{|c|c|c|}
\hline Item & Robot (n=22) & Open $(n=19)$ \\
\hline Symptoms & \multicolumn{2}{|c|}{$\mathrm{n}(\%)$} \\
\hline Flank pain & $14(63.64)$ & $9(47.37)$ \\
\hline Fever & $1(4.54)$ & $2(10.53)$ \\
\hline Haematuria & 0 & $1(5.26)$ \\
\hline Asymptomatic & $7(31.82)$ & $7(36.84)$ \\
\hline \multirow[t]{2}{*}{ Recurrent interval (years) } & \multicolumn{2}{|c|}{ median (range) } \\
\hline & $8(1-25)$ & $8(0.5-31)$ \\
\hline Etiologies for initial UPJO & \multicolumn{2}{|c|}{$\mathrm{n}$} \\
\hline Congenital & 13 & 8 \\
\hline Stone disease & 6 & 2 \\
\hline Vascular anomaly & 0 & 2 \\
\hline Etiologies for initial ureteral stricture & \multicolumn{2}{|c|}{$\mathrm{n}$} \\
\hline Stone disease & 3 & 6 \\
\hline Ureteral polyp & 0 & 1 \\
\hline Primary procedures & \multicolumn{2}{|c|}{$\mathrm{n}$} \\
\hline Open pyeloplasty & 9 & 6 \\
\hline Open uretero-ureterostomy & 1 & 5 \\
\hline Laparoscopic pyeloplasty & 5 & 4 \\
\hline Laparoscopic uretero-ureterostomy & 1 & 2 \\
\hline Open pyeloplasty + nephrostomy & 1 & 1 \\
\hline Open uretero-ureterostomy + balloon dilation & 1 & 0 \\
\hline Open pyeloplasty + endopyelotomy & 1 & 0 \\
\hline Open pyeloplasty + double $\mathrm{J}$ stent & 2 & 0 \\
\hline Laparoscopic pyeloplasty + nephrostomy & 1 & 0 \\
\hline $\begin{array}{c}\text { Laparoscopic pyeloplasty }+ \text { balloon dilation } \\
+ \text { endopyelotomy }\end{array}$ & 0 & 1 \\
\hline
\end{tabular}

3 
13

14

15

16

17

Peer) reviewing PDF | (2019:07:39011:1:1:NEW 15 Oct 2019) 
Table 2 (on next page)

Preoperative characteristics and postoperative outcomes of patients. 
Table 2.Preoperative characteristics and postoperative outcomes of patients.

\begin{tabular}{|c|c|c|c|}
\hline Item & Robot $(n=22)$ & Open $(n=19)$ & P value \\
\hline \multirow[t]{2}{*}{ Mean age (years) } & \multicolumn{2}{|c|}{ mean $\pm \mathrm{SD}$ (median) } & 0.760 \\
\hline & $37.82 \pm 18.56(36)$ & $36.16 \pm 15.54(39)$ & \\
\hline \multirow[t]{2}{*}{ Mean BMI (kg/m²) } & \multicolumn{2}{|c|}{ mean $\pm \mathrm{SD}$ (median) } & 0.969 \\
\hline & $22.75 \pm 3.65(22.48)$ & $22.79 \pm 2.94(22.86)$ & \\
\hline Gender & \multicolumn{2}{|c|}{$\mathrm{n}(\%)$} & 0.737 \\
\hline Male & $16(72.73)$ & $12(63.14)$ & \\
\hline Female & $6(27.27)$ & $7(36.86)$ & \\
\hline Side of disease & \multicolumn{2}{|c|}{$\mathrm{n}(\%)$} & $>0.999$ \\
\hline Left & $14(63.64)$ & $12(63.16)$ & \\
\hline Right & $8(36.36)$ & $7(36.84)$ & \\
\hline ASA & \multicolumn{2}{|c|}{$\mathrm{n}(\%)$} & 0.742 \\
\hline I & $5(22.73)$ & $4(21.05)$ & \\
\hline II & $15(68.18)$ & $14(73.68)$ & \\
\hline III & $2(9.09)$ & $1(5.27)$ & \\
\hline Procedure & \multicolumn{2}{|c|}{$\mathrm{n}(\%)$} & 0.145 \\
\hline Pyeloplasty & $19(86.36)$ & $12(63.16)$ & \\
\hline Uretero-ureterostomy & $3(13.63)$ & $7(36.84)$ & \\
\hline \multirow[t]{2}{*}{ Mean operative time (min) } & \multicolumn{2}{|c|}{ mean $\pm \mathrm{SD}$} & $<0.0001$ \\
\hline & $124.55 \pm 48.45$ & $185.11 \pm 49.71$ & \\
\hline \multirow[t]{2}{*}{ Mean blood loss (ml) } & \multicolumn{2}{|c|}{ mean $\pm \mathrm{SD}$} & 0.008 \\
\hline & $100.00 \pm 18.43$ & $182.60 \pm 23.89$ & \\
\hline Postoperative complications ${ }^{*}$ & & & \\
\hline Grade II & $2(9.09)$ & $7(36.84)$ & 0.057 \\
\hline Grade IIIa & 0 & $1(5.26)$ & 0.463 \\
\hline Grade IIIb & 0 & $1(5.26)$ & 0.463 \\
\hline \multirow[t]{2}{*}{ Median VAS } & \multicolumn{2}{|c|}{ median (range) } & 0.053 \\
\hline & $2.5(1-5)$ & $2(1-3)$ & \\
\hline \multirow[t]{2}{*}{ Cost (¥) } & \multicolumn{2}{|c|}{ mean $\pm \mathrm{SD}$} & $<0.0001$ \\
\hline & $61161.77 \pm 8567.67$ & $38470.79 \pm 9764.00$ & \\
\hline
\end{tabular}

BMI: Body mass index

ASA: American Society of Anesthesiologists Score

VAS: Visual analogue score

SD: Standard deviation

* Postoperative complications were classified using the Clavien-Dindo grading system. 
Table 3 (on next page)

Follow-up outcomes of patients. 
Table 3. Follow-up outcomes of patients.

\begin{tabular}{|c|c|c|c|}
\hline Items & $\operatorname{Robot}(n=21)$ & Open $(n=17)$ & $P$ value \\
\hline \multirow[t]{2}{*}{ Median follow up (month) } & \multicolumn{2}{|c|}{ median (range) } & 0.001 \\
\hline & $30(19-48)$ & $48(20-63)$ & \\
\hline \multirow[t]{2}{*}{ Success ${ }^{*}$} & \multicolumn{2}{|c|}{$\mathrm{n}(\%)$} & 0.775 \\
\hline & $18(85.71 \%)$ & $14(82.35 \%)$ & \\
\hline Outcomes & \multicolumn{2}{|c|}{$\mathrm{n}(\%)$} & \\
\hline Decreased hydronephrosis & $14(66.67)$ & $12(70.59)$ & - \\
\hline Stable hydronephrosis + symptom resolution & $4(19.05)$ & $2(11.76)$ & - \\
\hline Stable hydronephrosis + unrelieved symptoms & $3(14.28)$ & $1(5.89)$ & - \\
\hline Increased hydronephrosis & 0 & $2(11.76)$ & - \\
\hline
\end{tabular}

* Success was defined as a stable or decreased degree of hydronephrosis and absence of symptoms. 


\section{Figure 1}

Figure 1. Robot-assisted laparoscopic redo pyeloplasty for a 59-year-old male who underwent a failed left open pyeloplasty before.

(A) Modified $45^{\circ}$ lateral decubitus position for upper ureter stricture repair. Location of trocars was shown in this image. (B) The stricture segment was identified according to intraoperative morphological changes (dilated renal pelvis to normal ureter). (C) Excise the stricture segment. (D) Remove redundant renal pelvis and fibrosis segment. (E) Anastomosis is performed with fine interrupted suture. (F) Completed end-to-end anastomosis.
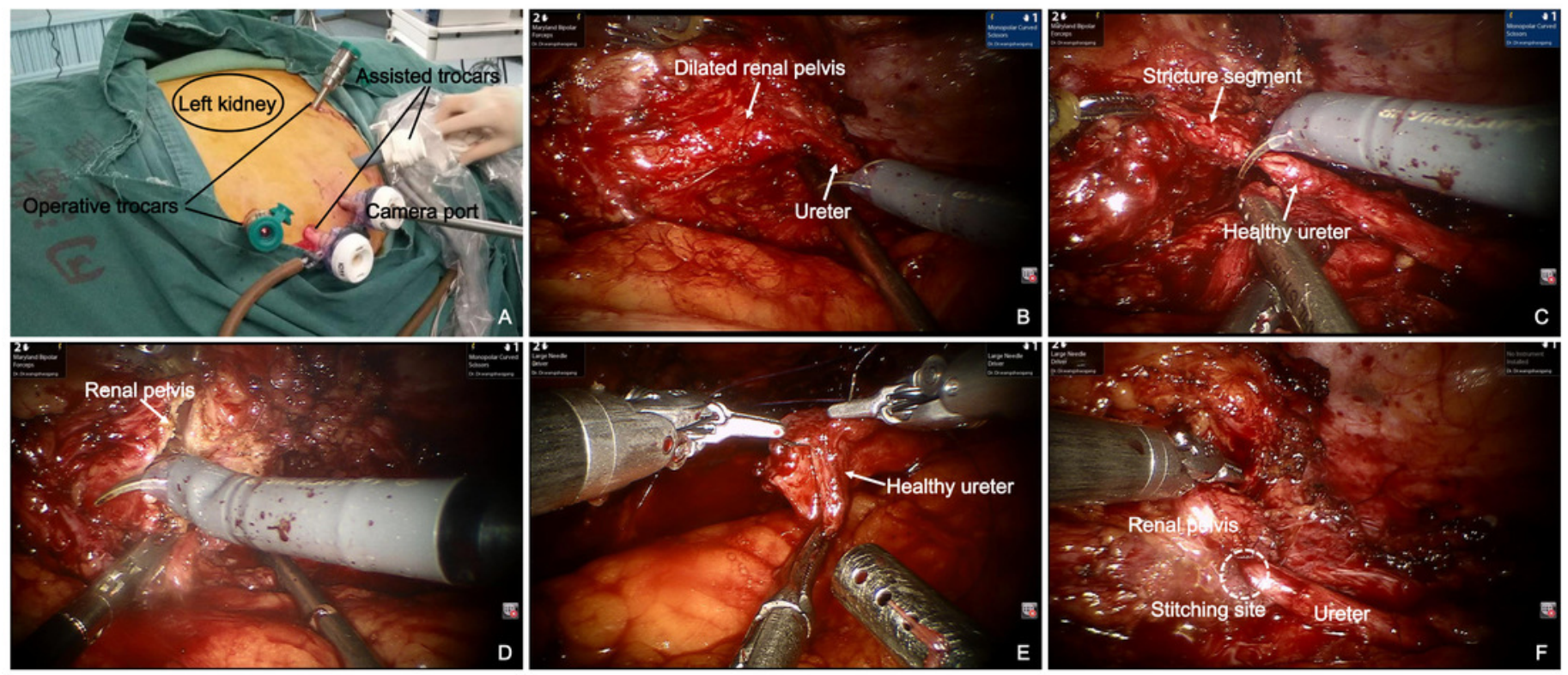